\title{
Una experiencia de Trabajo Social de grupo con pacientes con enfermedad mental grave y crónica. Una alternativa de recuperación psicosocial
}

\author{
An experience of Social group work with patients with serious \\ and chronic mental illness. An alternative of psychosocial recovery \\ Cristina Alonso SAlgado \\ Centro de Salud Mental de Hortaleza, Madrid \\ xtinaalonso@hotmail.com
}

Recibido: $14 / 02 / 2013$

Revisado: $10 / 04 / 2013$

Aceptado: 17/07/2013

Disponible on line: $27 / 06 / 2014$

\begin{abstract}
Resumen
Este articulo trata de mostrar una forma de intervención en grupo, a través de bajo la mirada de un trabajador social. Los participantes son personas con enfermedad mental grave y crónica, todos ellos incluidos en un programa de rehabilitación psicosocial y con seguimiento terapéutico en lo que denominamos continuidad de cuidados. Los protagonistas muestran relatos y testimonios personales en su manera de vivir y sentir la enfermedad mental.

Una intervención grupal que rescata dificultades en la relación social y focaliza la mirada profesional en cómo potenciar y compartir en grupo recursos para abordarlas.

Esta intervención de trabajo social se ha realizado en un centro de salud mental, ambulatorio y público, de la Comunidad de Madrid.

Con una metodología que trata de poner voz a los testimonios individuales de pacientes a través de textos escritos. En dichos textos, se recuperan y transmiten vivencias, historias, «sus historias» con modos de expresión diferentes y particulares. Experiencias que potencian no solo, la recuperación de la propia imagen e identidad sino la relación y representación de la enfermedad mental en la sociedad.

Palabras clave: experiencia de trabajo social con grupos, enfermedad mental severa y crónica, rehabilitación psicosocial, continuidad de cuidados, testimonios personales.
\end{abstract}

\begin{abstract}
This article tries to show a way of group intervention, under the regard of a social worker. Participants are people with chronic and severe mental illness, all of them included in a program of psychosocial rehabilitation and therapeutic monitoring, known as continuity of care. The protagonists show stories and personal testimonies in their way of living and feeling mental illness. A group intervention which rescues difficulties in social relationships and professional look focuses on enhancing expressive capabilities as well as on sharing resources in group to address them.

This intervention of social work has been done in a public outpatient mental health center in the Madrid region. The methodology tries to put a voice to the individual patient testimonials through written texts. In these texts, experiences and stories, «their stories», are recovered and transmitted from various and particular ways of expression. Experiences that enhance not only the recovery of self-image and identity, but the relationship and representation of mental illness in society.
\end{abstract}

Keywords: social group work experience, severe and chronic mental illness, psychosocial rehabilitation, continuity of care, personal testimonies.

Referencia normalizada: Alonso Salgado, C. (2014): «Una experiencia de Trabajo Social de grupo con pacientes con enfermedad mental grave y crónica. Una alternativa de recuperación psicosocial». Cuadernos de Trabajo Social, 27(1): 223-231.

Sumario: Introducción. 1. El grupo desde la perspectiva del Trabajo Social. 2. Metodología y diseño del grupo. 3. El grupo como alternativa de recuperación psicosocial. 4. Los protagonistas: los pacientes. 5. Desarrollo del grupo. 6. Conclusiones. 7. Referencias bibliográficas. 


\section{Introducción}

El Trabajo Social en salud mental ha experimentado cambios significativos en los últimos 30 años. Con la reforma psiquiátrica, iniciada en España en la década de 1980, comienza un periodo que proporciona las condiciones necesarias para abordar la atención a la enfermedad mental a través de una filosofía comunitaria y un modelo de tratamiento integral, con unos principios que - como expresa Desviat (1994) - exigen un sistema de salud universal, equitativo, descentralizado y participativo.

El Trabajo Social, predecesor en esta metodología de intervención comunitaria, rebasó profesionalmente el ámbito sanitario-psiquiátrico de la enfermedad para dedicarse plenamente a desarrollar las dimensiones social, psicosocial y de promoción de los derechos sociales de los enfermos mentales.

Garcés (2010) expresa que durante años la figura del trabajador social había sido ambigua, y consiguió ir evolucionando poco a poco. Así, los trabajadores sociales psiquiátricos hemos ido renovando nuestras intervenciones, en paralelo con el resto de profesionales de los equipos multiprofesionales; hemos ido aprendiendo a identificar los problemas, a definirlos y a proponer actuaciones muy variadas, dentro de las líneas de intervención terapéutica incorporadas en un sistema institucional y organizativo.

Con el tiempo hemos ido desempeñando diferentes papeles y ocupando funciones, adaptándonos a las características del recurso o al lugar de intervención; entendiendo nuestra especificidad e identificando nuestra tarea en salud mental, entre otras funciones, en lo que define Ituarte (1992, p. 11) como trabajo social clínico, reconociendo las intervenciones individuales conjuntamente con las situaciones sociales, combinando aspectos psicoterapéuticos con intervenciones contextuales. Así, durante los últimos 20 años, la intervención asistencial y terapéutica llevada a cabo por los equipos profesionales de salud mental ha ido contemplando, en los diferentes proyectos terapéuticos, no solo síntomas sino discapacidades, déficits sociales y las propias dificultades que conlleva vivir en sociedad con una enfermedad mental.

Entre los objetivos, de cualquier proceso de rehabilitación, figuran el de tratar de alcanzar y mantener un grado de autonomía y de control de las situaciones de conflicto, y de reajustar un funcionamiento social autónomo, así como el de conseguir una forma de vida lo más satisfactoria posible.

La recuperación - según define Lieberman (2004) - incluye una remisión de síntomas, un funcionamiento ocupacional, una vida independiente y el mantenimiento de relaciones sociales y de amistades con quienes compartir actividades de manera regular.

Los objetivos, en esta intervención grupal de trabajo social, se plantean dos direcciones: por un lado, el desarrollo de capacidades individuales de los participantes y, por el otro, la participación en el entorno social, la participación ciudadana a la que hace referencia Sarraceno (1999).

La coyuntura profesional y el momento social en los equipos de salud comunitarios, desde su implantación en Madrid, han posibilitado que en el Trabajo Social se hayan podido sostener experiencias como las del grupo que se describe en este texto. En el momento actual y con el nuevo modelo sanitario en el que prima la gestión de la eficiencia de recursos y el intento de separación de lo sanitario y no sanitario, con una valoración de las intervenciones terapéuticas, con criterios casi exclusivamente económicos, quizás esta experiencia ya no sería posible. La proyección de la salud mental actual corre peligro de volver a escindir, en los proyectos terapéuticos, lo sanitario de lo social, como si en el sujeto no fuera un todo su mejoría de síntomas y su adaptación al entorno social.

\section{El grupo desde la perspectiva del Traba- jo Social}

Nadie llega a convertirse en humano si está solo: nos hacemos humanos los unos a los otros (Fernando Savater, 1999)

Si la participación en grupo es enriquecedora para cualquier individuo, para los pacientes con trastorno mental grave es además rehabilitadora, e indispensable en su proceso de adaptación a la manera de vivir con la enfermedad. El grupo proporciona y favorece la participación en la vida social y contribuye a reducir la tendencia al aislamiento. «Todo el mundo tiene su hueco o debería tenerlo y luchar por 
mantenerlo. El tener un espacio donde poder crear un entorno, en el que sentirse a gusto y poder desarrollarse, es fundamental para una buena calidad de vida» (Texto de F.G. Paciente y Participante del grupo, 2010).

A partir de la premisa de que los elementos centrales de cohesión y comunicación entre las personas y en las diferentes culturas son la lengua y la religión, este grupo se aglutina en torno a la tarea de expresar, a través del lenguaje y de textos escritos, las diferentes formas de vivir y manifestar la dificultad de sentir y vivir en la sociedad con una enfermedad mental.

Los textos, relatados en primera persona por pacientes con trastorno mental grave, ofrecen a los profesionales y al resto de los componentes del grupo la posibilidad de mirar, escuchar y ser testigos de experiencias singulares; en ocasiones las experiencias de los participantes son cercanas entre sí, temporal y espacialmente, aunque lejanas en lo referente a actuaciones, vivencias y realidades.

Cuando te aqueja una enfermedad crónica, que te obliga a abandonar todas las actividades que se hacen en la vida normal, se ha de buscar un nuevo sentido donde la enfermedad ha de ser el mero eje. Para muchos ese nuevo sentido es aprender a convivir con la enfermedad, asumirla y adoptar lo que te permite hacer con lo que idealmente desearías. Para otros el nuevo sentido es precisamente no tener sentido y dejarse ir a la deriva, sin objetivos ni ilusiones. Para mí, el sentido de la vida, ahora, es adquirir conocimientos sobre mi propia vida que surgen tras asumir las limitaciones que me ha dado la enfermedad (Texto J.A.R. paciente y participante del grupo, 2010).

\section{Metodología y diseño del grupo}

El nacimiento del grupo surge como una alternativa y estrategia a una demanda de insatisfacción individual, común y generalizada.

Demanda sobre todo referida a las quejas en las relaciones de convivencia y a la insatisfacción por las escasas medidas de apoyo del sistema, social, laboral, político, de pensiones y ayudas económicas, así como de soportes sociales en vivienda y, en general, por todo lo referente al sufrimiento social que arrastra el trastorno mental crónico.

El diseño parte, por lo tanto, de una demanda individual que se trabaja en grupo, que lo va conformando, a lo largo del tiempo y de compartir las lecturas de los textos elaborados individualmente. Con un encuadre de grupo abierto, voluntario y variable en el número de participantes, que oscila entre 12 y 15 pacientes, incorporados en general al programa de rehabilitación y continuidad de cuidados durante varios años.

La continuidad de cuidados es un modelo en salud mental que pretende una atención integral de las necesidades de los pacientes con trastorno mental grave. Su intervención tiene lugar tanto en el servicio de salud mental, como en el entorno vital.

Se trata de un grupo de intervención de frecuencia semanal, de una hora y media de intervención e instituido como una actividad estable entre las acciones terapéuticas de rehabilitación del centro de salud mental

La elección del tema grupal la propone cualquier participante, unas veces de manera consensuada entre todos, otras veces responde a cualquier emergencia o vivencia de los participantes y surge por algún suceso o acontecimiento social significativo: violencia ciudadana en el barrio, atentados en la ciudad o en el distrito; o por situaciones de dinámica interna, como internamiento de algún participante, duelos etc.

El tiempo del grupo transcurre, primero, con la escritura individual del texto sobre el tema elegido, le sigue la lectura que realizada cada uno de los participantes, y que escucha el resto del grupo y, por último, se archiva el texto en cada una de las carpetas personales de los miembros del grupo.

La asiduidad de los participantes al grupo varía según los momentos y las personas; muchos de ellos han sido muy constantes, en otros ha fluctuado su interés por la participación en el mismo y han asistido de una manera discontinua o esporádica.

\section{El grupo como alternativa de recupera- ción psicosocial}

\section{Con la palabra el hombre se hace Hombre} (Freire, 2003)

Para una prevención terciaria de la salud mental, Caplan (1985) aporta una serie de medidas destinadas a reducir el deterioro social de 
una comunidad y propone las intervenciones de trabajo grupal como un espacio que favorece los valores de participación y de tolerancia. El grupo se fundamenta en el respeto de las opiniones diferentes, en potenciar el derecho a la palabra como el instrumento genuino del Trabajo Social, según palabras de Hernández Aristu (2002); como un lugar para crear vínculos sociales, promover nuevas realidades y rescatar diferentes roles sociales. Con un diseño de grupo abierto al número de participantes, sin limitación de tiempo de su permanencia, participativo y libre sobre los temas a compartir, que son elegidos libremente, salvo el relativo a intentar no hablar directamente de los síntomas de la enfermedad para los que - se ha consensuado - existen ya otros espacios de comunicación.

$\mathrm{Su}$ objetivo general es constituir un grupo con las premisas planteadas en cualquier proceso de recuperación psicosocial: rescatar aspectos positivos, capacidades e ilusiones en los participantes. «Una acción organizada con fines educativos que promueve al ser humano por medio de la participación grupal, proporcionándole el sentimiento de ser miembro de la sociedad a la que pertenece» (Contreras de Wilhelm, 2003).

Una participación activa en el propio proceso terapéutico contribuye a la lucha contra los estereotipos culturales sobre la discapacidad del enfermo mental, en la que estamos todos inmersos: pacientes, profesionales y el entorno social; ya que todos somos y estamos sumidos en la misma cultura que estigmatiza la enfermedad mental (Estudio sobre Estigma, 2006).

Se propone esta forma de intervención grupal con el objetivo general y primordial de mejorar el funcionamiento social de las personas participantes. Se debe tener en cuenta, además del diagnóstico clínico y sus síntomas, otros aspectos y situaciones personales, diferentes condiciones de vida, de procesos afectivos, emocionales, intelectuales, laborales y las diferentes circunstancias vitales, de cada sujeto participante, que dificultan o incapacitan para la participación social, mediante un espacio facilitador de intercambio y comunicación de dichas dificultades

La finalidad general del grupo, como de cualquier otra actividad rehabilitadora de salud mental comunitaria, es tratar de conseguir mantener a las personas en su medio habitual, minimizar los déficits y las carencias, así como habilitar y recuperar aquellas capacidades sociales para el manejo de la enfermedad, además de procurar el mayor bienestar en la convivencia social

Este grupo, por lo tanto, se conforma con una unión de individualidades con diferentes niveles de conciencia de enfermedad, y niveles culturales, peculiaridades, etc., que comparten un espacio común, acompañados y reconducidos por un trabajador social cuya tarea primordial es conseguir la participación y el respeto de todos y de todas las opiniones; el reconocimiento de las singularidades; la aceptación de las dificultades de comunicación y el poder compartir la presión social que supone, especialmente para algunos participantes, un diagnóstico de enfermedad mental crónica, que estigmatiza e incapacita para una vida social normalizada

Cómo nos ven los demás. Los enfermos mentales somos gente a los que la sociedad discrimina. Si una persona se rompe una pierna la gente le pregunta: ¿Qué tal estas? Sin embargo a los que padecemos una enfermedad psíquica nos tratan que parezca que no tenemos nada y por ello algunas personas se creen que le echamos cuento para no trabajar. Existe también cierto morbo entre algunas personas hacia las enfermedades de este tipo, debido a un desconocimiento. En mi caso, si no digo nada de mi enfermedad, nadie se entera, funciono con una normalidad profunda, cuando se lo cuento a alguien que lo desconoce, se extraña. Con la enfermedad se pasa muy mal y salvo raras excepciones nadie tiene en cuenta esto (Varón, 62 años, soltero, vive con su madre viuda. En tratamiento desde 1990, diagnosticado de esquizofrenia paranoide).

Esta dinámica profesional de funcionamiento pretende el cuidado de los participantes con sus diferentes ritmos y momentos, sin prejuicio de comportamientos o actitudes; vela por un funcionamiento casi autogestionado, con la exclusiva función profesional de potenciar, coordinar y sostener un espacio que promueva la confianza de cada uno de los participantes con el resto del grupo. Se consigue que algunos de sus participantes expresen, en sus textos, emociones y pensamientos que no consiguen articular en su comunicación verbal. 
El miedo. Los miedos de las personas surgen tras alguna experiencia negativa que no se puede superar; o bien los_miedos internos, tras sufrir alguna sensación de no poder_controlar una situación. Creo que el miedo pasa al inconsciente y luego al revivir la situación, vuelve a producir angustia.

Parece ser que el miedo a lo desconocido es un tipo especial de miedo porque anula la capacidad de raciocinio, no se sabe qué hacer, puesto que no se advierte qué es lo que produce la sensación tan angustiante.

Siento que mi vida es un viaje en el que constantemente debo avanzar y retroceder, alternando dos conceptos, la emoción y la razón. Esto es para mí la esencia de la enfermedad mental pues la sensación de pérdida que se apodera del ser cuando tal transformación ocurre puede ser tal que parece constituirse en una amenaza muy concreta de perder la orientación vital, de no saber quién es uno mismo (Varón, 47 años, soltero. En tratamiento desde 2000, diagnosticado de esquizofrenia paranoide. Actualmente realiza estudios universitarios)

\section{Los protagonistas: los pacientes}

Qué es la locura, a veces es difícil definir la locura, por ello suele hacerse la definición de cordura. Para unos la locura es un extravío de la mente que empieza a fantasear y a confundir la realidad con la imaginación; pero para otros tal distinción no es fácil de hacer, por lo cual lo que para unos es locura para otros es creatividad. Y efectivamente la creatividad artística parece tener una parte de locura al apartarse de lo normal o lo habitual. Hay personas que son creadores y son considerados locos porque parece que hacen las mismas cosas que los locos y hablan como ellos (Texto de J.A.R., 2010).

Una de las poblaciones diana de los centros de salud mental son las personas con trastorno mental grave y crónico, categoría que incluye a las personas que reúnen, en si mismas una serie de requisitos en relación al diagnóstico y al tiempo de evolución de la enfermedad según documento de consenso (Ministerio de Trabajo y Asuntos Sociales, 2003).

El grupo constituido esencialmente por pacientes psicóticos de largos años de evolución de enfermedad, algunos con tratamiento simultáneo en salud y en recursos de la red de atención social a la enfermedad mental grave y cró- nica de la Consejería de Asuntos Sociales. La distribución por sexos coincide con las estadísticas generales de los programas terapéuticos de pacientes crónicos: una población en su mayoría varones, el 65 por ciento están solteros/as; el 90 por ciento conviven con sus familias de origen; con buena adherencia al tratamiento; con buena aceptación de su condición de enfermo mental; en general con certificado de minusvalía, algunos con incapacidad laboral, generalmente perceptores de la pensión no contributiva, con una media de ingresos de 350 euros, fumadores; muchos de ellos con residencia cercana al Centro de Salud. En general, son pacientes con importantes deficiencias en su vínculo social y en consecuencia con problemas de aislamiento.

Las dificultades y quejas más generalizadas de los participantes — como ya hemos referido- se concentran en la escasa capacidad económica para poder acceder a una vida independiente; un porcentaje, también alto, plantea problemas de convivencia con el entorno y en las relaciones sociales cotidianas, especialmente en el ámbito familiar.

Respecto a su forma de vida, algunos participantes han conseguido vivir de manera independiente, autónoma, aunque con apoyos profesionales; la independencia, generalmente, más que conseguida se la ha impuesto el fallecimiento de sus progenitores; otros conviven en contextos institucionales y en todos coincide su buena adherencia al tratamiento y la voluntad en mantener seguimientos terapéuticos. En general, dichos participantes valoran la posibilidad de poder expresar sus opiniones, sus inquietudes y hasta sus delirios, normalizando, en cierta medida, los discursos y la sintomatología que supone la enfermedad mental.

Sus relaciones con el entorno social, externas al Centro de Salud mental, son muy escasas, quedando casi reducidas a su circuito terapéutico, a los profesionales de referencia de salud: mental o primaria, y de los servicios sociales. Así, fuera del contexto profesional, la vida social se reduce casi exclusivamente a los familiares más cercanos; el potenciar el trabajo en grupo y las relaciones sociales entre iguales supone, en la mayor parte de los casos, un enriquecimiento en su vida social y relacional, como apunta Sarraceno (1999) «Todo proceso de autoidentificación de una minoría es un proceso enriquecedor». 
Casi todos los participantes coinciden en que están mucho más ocupados y preocupados por aspectos relacionados con su enfermedad, sus síntomas y sus dificultades, que en realizar deseos, proyectos e intereses relacionados con la vida.

Dependiendo de las circunstancias, algunas imponderables como las mermadas pensiones económicas que les sitúan como personas con rentas económicas muy bajas, se trata de sujetos que han logrado una vida más o menos sosegada, ordenada y participativa en el entorno terapéutico, aunque en el entorno comunitario su participación es deficitaria y los progresos sociales que se consiguen son muy lentos y escasos.

En general, mantienen un seguimiento multiprofesional integral, es decir: terapéutico, farmacológico y social. En su plan terapéutico participan diferentes profesionales del equipo y la continuidad de su seguimiento, enunciada anteriormente como "continuidad de cuidados», está coordinada por los profesionales no facultativos entre los que se encuentran los trabajadores sociales.

La población a la que nos referimos es, por lo tanto, una conjunto de personas, en su mayoría con un diagnóstico de psicosis, de largos años de evolución y de tratamiento prolongado. Como se define uno de los protagonistas del grupo:

La identidad, pregunta difícil de contestar cuando te aqueja una enfermedad crónica, que te obliga a abandonar todas las actividades que se hacen en la vida normal. Se ha de buscar un nuevo sentido donde la enfermedad ha de ser el mero eje. Para muchos ese nuevo sentido es aprender a convivir con la enfermedad, asumirla y a adoptar lo que te permite hacer con lo que idealmente desearías. Para otros el nuevo sentido es precisamente no tener sentido y dejarse ir a la deriva, sin objetivos ni ilusiones. Para mí el sentido de la vida es adquirir más conocimientos sobre la propia vida que surgen tras asumir las limitaciones que me da la enfermedad (Texto de J.A.R. paciente y participante del grupo, 2011)

\section{Desarrollo del grupo}

No hay hombre absolutamente inculto: el hombre se humaniza expresando $y$ diciendo su mundo (Paulo Freire, 2003)

Se va incorporando al grupo la utilización del diccionario de lengua castellana de la Real
Academia Española, como una herramienta más que favorece la comprensión del lenguaje y sus significados entre los componentes que se distinguen por su diferente edad, nivel cultural, intereses, etc.

En todas las sesiones de este espacio de grupo semanal, se han tratado de potenciar relaciones de confianza, de seguridad, de respeto y se han conseguido textos que expresan aspectos personales y diferentes historias, que rememoran situaciones y vivencias, en diferentes contextos y situaciones, como expresa Rubens R. Méndez (2008). La historicidad para el Trabajo Social significa introducir a estas personas dentro de un sistema de pertenencia social, familiar, cultural, institucional, de relaciones desde donde la persona es significada y a su vez resignificada su realidad circundante.

Se consigue, así, crear opinión en todos y en cada uno de sus participantes y no solo en los más comunicativos.

La Pereza. Yo estoy casi permanentemente en un estado de pereza tanto física como intelectualmente. Ello es debido a que noto en $\mathrm{mi}$ interior un cansancio vital, que no se a que atribuirlo. Normalmente me cuesta bastante trabajo empezar a hacer cualquier actividad y suelo empezar con mucho ímpetu, y a veces, lo abandono después (J.G., varón, 47 años, soltero. En tratamiento desde 2000. Diagnosticado de esquizofrenia paranoide. Actualmente realiza estudios universitarios).

A través de los textos, se muestran cuestiones muy diferentes, unas de índole personal, emocional; y otras, relativas a opiniones, pensamientos, actitudes, dificultades, etc. Los temas más recurrentes son: el tiempo, la preocupación por el futuro, el miedo, la pereza, la soledad, la identidad subjetiva, la identidad social, las relaciones en el entorno social y familiar y la convivencia con la enfermedad.

La discapacidad. En la palabra persona discapacitada prima lo segundo sobre lo primero y debería ser al revés. Los medios de comunicación desinforman.

El trabajo. Es a la vez un determinante y un resultado. Saca al enfermo mental del rol de enfermo y lo lleva al_rol de normalizado, en el que adquiere por un lado autoestima, sentimiento de utilidad, relaciones sociales y le libra de la ex- 
clusión social y por otro lado le da independencia económica, autonomía personal y social.

Es, por tanto, a su vez un determinante y un resultado social, económico y laboral. Por todo ello es la terapia más completa que existe para evitar el rechazo y exclusión social a la que la sociedad, que se encuentra enmarcada en un ámbito socio laboral y productivo tiende en este momento. Es el momento en que el paciente se convierte en el protagonista de su propia rehabilitación y de su tiempo y deja de ser un mero espectador, que deja pasar los acontecimientos de la vida. La exclusión y el estigma del enfermo mental, solo se observa en la convivencia con él. Es ahí donde se ve el desajuste y la verdadera discapacidad (Varón, 42 años, soltero, vive con su madre, viuda. En tratamiento desde 1995. Diagnosticado como trastorno bipolar; con experiencias laborales en puestos de responsabilidad).

El tiempo. Una de mis principales preocupaciones son las expectativas de futuro. Me pregunto qué será de mí dentro de unos años, cuando esté solo. Ahora vivo bien, pero no sé si seré capaz de valerme por mí mismo y salir adelante, llevando una vida normal Otra preocupación es el sentimiento de inutilidad que me invade. Siento que cada vez hago menos cosas y que paso mucho tiempo sin hacer nada, por lo que me atrofio. Entre mis fantasías no abandono la posibilidad de compartir mi vida en pareja aunque siento desesperanza y temor al futuro.

La libertad. Para mí es un valor fundamental la libertad, aparte de la cuestión económica que también tiene su importancia, valoro mucho la buena relación con mi familia, mis padres y mi psiquiatra. También valoro mucho la seguridad personal.

Independencia y autonomía. Ser independiente significa saber tomar decisiones por uno mismo; no estar bajo la ayuda o la decisión de otros, poder adoptar posturas sin que otros nos influyan. Yo soy independiente

Ser autónomo supone que en un momento dado somos capaces de emanciparnos, de buscar caminos sin que otros estén inmersos, elegir con plena libertad (Mujer, 60 años, soltera vive con hermana. En tratamiento desde 1980. Profesora de Literatura y Lengua en Instituto Público. Jubilada. Diferentes diagnósticos psiquiátricos: esquizofrenia paranoide, trastorno bipolar).
Libertad, bonita palabra. La libertad es un estado mental que consiste en la calidad de la que cada uno piensa. La libertad es la capacidad de poder volar pero en este sistema te las suelen cortar con facilidad (las alas) por cualquier acto que se haga fuera de la ley, encerrándonos en una institución, aunque al ser un estado mental te puedes sentir libre estés donde estés, pocas son las personas que se sienten libres presos de su trabajo, de su centro de día o de la cárcel libertad de expresión y pensamiento. Te puedes expresar sin dañar a otra persona siendo muy difícil porque todos estamos de acuerdo con lo que se expresa. La libertad de cada uno empieza donde acaba la nuestra, ¿hay libertad?

Si todas las personas estuvieran en otro tipo de sistema. Si hay prisiones es porque algo falla y si algo falla no hay libertad; Puedes ser libre con un libro y al cerrarlo preso en la geometría del hormigón; puedes ser libre con el viento con la escucha de los pájaros o con el amanecer pero cuando se acaba, siempre queda un sentimiento una carga de energía que se llama libertad (Varón, 36 años. Soltero vive en piso tutelado. En tratamiento desde 2002, diagnosticado de esquizofrenia paranoide. Madre viuda. Escribe casi siempre en rima).

\section{Conclusiones}

La transmisión de la experiencia de este grupo, la presentación de alguno de los textos de sus participantes y el relato de testimonios de los propios protagonistas pretenden contribuir a la mejora de la imagen y la representación social de quiénes son y cómo revelan su realidad algunas personas afectadas de una enfermedad mental grave y crónica. La intención es mostrar una mirada diferente, positiva y esperanzadora de los procesos de integración, de algunos de sus valores y capacidades.

El grupo ha conseguido que personas con el rol determinante de «paciente» se sitúen en una realidad diferente a la de ser enfermo mental exclusivamente. A lo largo del grupo se han señalado y compartido emociones, como la pereza, la inhibición, el miedo al futuro y se han podido rescatar deseos y proyectos.

Desde la perspectiva del Trabajo Social, este grupo con pacientes crónicos ha supuesto una nueva forma de intervención y de procurar nuevos retos de participación profesional; ha supuesto un contexto de participación activa, en el propio proceso de recuperación, en perso- 
nas, con diagnóstico de enfermedad mental grave y crónica.

Se han puesto en práctica valores del trabajo social como: procurar bienestar y potenciar el respeto a la diferencia; el conseguir una participación activa de los propios usuarios en los objetivos de su rehabilitación; la reflexión y el reconocimiento de las propias capacidades y dificultades

Desde la perspectiva de rehabilitación, el trabajo en grupo ha supuesto explorar capacidades de creatividad y de empatía con el resto de miembros del grupo

Se ha inculcado el respeto y el derecho de los pacientes no solo, a participar activamente en su proceso de rehabilitación sino a poner en práctica el derecho de opinión y de expresión como ciudadanos

Se han trabajado valores de la rehabilitación social como la constancia en la actividad; el respeto a las diferencias y a los ritmos de aportación aceptando las dificultades y hasta la autoexclusión temporal de alguno de sus participantes

Se ha fomentado el cuidado de un espacio y un tiempo de grupo dando importancia y atención al propio material elaborado.

En general, personas muy desautorizadas e invalidadas en su medio habitual ya sea por su diagnóstico o por su papel social, casi exclusivo, de enfermo mental, el grupo y sus textos les ha permitido expresar y proyectar otra realidad, rescatar otros aspectos de sí mismos El discurso, singular, de cada uno de los participantes, a veces delirante, ha supuesto una cierta normalización del lenguaje disgregado, incoherente, en general poco aceptado fuera del contexto psiquiátrico y terapéutico Es evidente que la lectura de algunos de los textos aquí transcritos contradice ciertos discursos psiquiátricos que estigmatizan y vinculan la enfermedad mental con la peligrosidad o la falta de capacidades para vivir en sociedad

En conclusión, la experiencia de grupo ha supuesto una relación que ha fomentado vínculos afectivos entre sus miembros y ha rescatado cualidades y valores entre sus protagonistas. Apunta Arija (1998): «El grupo es un inmenso potencial para que las personas rescaten la viveza que proporciona lo colectivo. Apegarse a la vida enraizando con otros en la ayuda mutua».

La motivación en pacientes donde la pereza y la falta de proyecto vital es una constante ha supuesto un esfuerzo añadido, a la coordinación del grupo, el conseguir un ambiente positivo de trabajo personal, así como la obtención de los objetivos grupales previstos

Por lo tanto, las dificultades en la realización de este grupo han estado enmarcadas, especialmente, en la población de intervención y su psicopatología que, en momentos, han condicionado la dinámica y el desarrollo del mismo

Comparto el deseo que manifiesta Belén Arija,( 2004) de animar, a los compañeros de profesión, a realizar intervenciones con grupos que contribuyen a desarrollar las propias capacidades, la propia creatividad personal y combinar las propias motivaciones con las de los usuarios enriqueciendo los procesos de acompañamiento social.

Mi agradecimiento, especialmente, a los participantes del grupo por la valentía en sus aportaciones, su generosidad en permitir la utilización de sus textos y el enriquecimiento que suponen sus discursos, elocuentes, sensibles y más reflexivos de lo esperado.

«Vivimos dentro de historias, no dentro de estadísticas» (Gilberto, 2002).

\section{Referencias bibliográficas}

Alonso, C. (2005). Los efectos sociales de la enfermedad mental. Revista Trabajo Social Hoy, 46, 19 a 30.

Arija, B. (2004). Un proceso de vinculación a través del arte. Trabajo Social con grupos. Revista Trabajo Social Hoy. [Monográfico de Intervención Social con Grupos] 90-113.

Arija, B. (2012) Acompañamiento con grupos en Trabajo Social. Un modelo interfocal para la comprensión y sistematización de la praxis. Cuadernos de Trabajo Social, 25, 427-438.

Bravo, MF., Fernández Liria, A. y Alonso. M (2004). Origen y desarrollo de los programas de seguimiento y cuidados para pacientes mentales graves y crónicos en la comunidad. Revista $A E N$, 92, 25-51.

Caplan, G. (1985). Principios de Psiquiatría Preventiva. Buenos Aires: Paidós. 
Contreras de Wilhelm, Y. (2003). Trabajo Social de grupos. México: Pax- México

Desviat, M. (1994). La Reforma Psiquiátrica II. Madrid: Ediciones Dor ,S.L.

Documento de consenso (2003). Rehabilitación e integración social de las personas con trastorno mental grave (2003). Madrid: Ministerio de Trabajo y Asuntos Sociales.

VV.AA. (2006) El estigma de la Enfermedad Mental. Madrid: Consejería de Familia y Asuntos Sociales, Universidad Complutense de Madrid y Caja Madrid

Freire, P. (2003) Pedagogía del Oprimido. México: Siglo XXI.

Garcés, E. (2010). Trabajo Social en Salud Mental Cuadernos de Trabajo Social, 23, 333-352.

García Nieto, R., De La Torre Brasas, F. ya Cantero Fernández (2004). Aplicación de la terapia psicológica integrada a pacientes psicóticos crónicos en un centro de salud mental. Revista AEN - Madrid 9, 81- 96 Madrid

Gómez Esteban, R., Martín Vázquez Mª J., González López A., Flores Álvarez M., Bote Bonaechea B. y Güerre Lobera $\mathrm{M}^{\mathrm{a}} \mathrm{J}$. (2003). Reflexiones con pacientes esquizofrénicos Revista de la AEN 88,17-33.

Henry, East y Schimtz (coords) (2004) Trabajo Social con Grupos Modelos de intervención (pp. 121-234). Madrid: Narcea.

Ituarte, A. (1992). Procedimiento y Proceso en Trabajo Social Clínico. Consejo de Trabajadores Sociales. Madrid: Siglo XXI.

Liberman, R. y Kopelwicz, A. (2004) Un enfoque empírico de la recuperación de la esquizofrenia: definir la recuperación e identificar los factores que pueden facilitarla. Rehabilitación Psicosocial, 1,12-29.

Miranda Aranda, M. (2008). Historia e identidad del Trabajo social en Salud. Revista Trabajo Social y Salud, 60, 7-21.

Olza Zubiri, M. y Hernández Aristu, M. (2002) Trabajo Social, cuestiones sobre el que y el como. Teoría y Practica del Trabajo Social, 1, 9-26 y 67-85.

Rubens R. Méndez (2008). La Práctica del Trabajo Social y el despliegue de sus condiciones de posibilidad en el campo de la Salud Mental. Trabajo Social y Salud, 59, 7-78.

Rubens, R. y cols. (2008). La invención de la intervención en el campo de la salud mental Revista Trabajo Social y Salud. 59, 24 -36.

Sarraceno, B. (1999). La ciudadanía como forma de tolerancia. (Congreso Catalán de Salud Mental). Barcelona.

Zamanillo, T. (2008). Trabajo Social con grupos y pedagogía ciudadana. Madrid: Síntesis. 